\title{
A synthetic strategy for carbon nanospheres impregnated with highly monodispersed metal nanoparticles
}

\author{
Tianyu Yang ${ }^{1,6}$, Huajuan Ling ${ }^{2,6}$, Jean-François Lamonier ${ }^{3}$, Mietek Jaroniec ${ }^{4}$, Jun Huang ${ }^{2}$, Michael J Monteiro ${ }^{1}$ \\ and Jian Liu ${ }^{1,5}$
}

$\mathrm{N}$-doped mesoporous carbon nanospheres (N-MCN@M) impregnated with uniformly dispersed noble-metal ( $\mathrm{Au}, \mathrm{Pt}, \mathrm{Rh}, \mathrm{Ru}, \mathrm{Ag}$, $\mathrm{Pd}$ and Ir) nanoparticles are rationally designed and synthesized for hydrogenation reactions. This facile and generally applicable synthetic strategy ensured confinement of the noble-metal nanoparticles within different carbon morphologies, including mesoporous spheres, hollow particles and core-shell particles. High loading of the noble-metal nanoparticles from 8 to $44 \%$ was accomplished by tuning the initial concentration of metal salts. Even at very high loadings ( $>40 \mathrm{wt} \%$ ), a homogeneous dispersion of uniform metal nanoparticles throughout the carbon nanostructures was achieved. The proposed synthesis is also well suited for the fabrication of carbon spheres loaded with bimetallic nanoparticles (AuPt, AuRh and PtRh). Examination of these metal-loaded carbon particles as catalysts for the hydrogenation of benzaldehyde gave $100 \%$ selectivity toward carbonyl group at room and higher reaction temperatures. The outstanding performance of Au nanoparticles gave an unprecedented turn over frequency 2-4 times greater than those of Pt nanoparticles with the same size, loading and support.

NPG Asia Materials (2016) 8, e240; doi:10.1038/am.2015.145; published online 5 February 2016

\section{INTRODUCTION}

Metallic and bimetallic nanoparticles with controlled shape, size and composition have attracted considerable attention because of their excellent catalytic performance in refining petroleum compounds, organic transformations, hydrogen production and many other important chemical reactions. ${ }^{1-4}$ Among them, gold nanoparticles are powerful catalysts for aerobic oxidative processes, but considered as unfavorable for hydrogenations because of their low hydrogendelivery rates. Agglomeration of gold and other metal nanoparticles under conventional reaction conditions (for example, high temperature, high pressure, strong acidic or basic conditions) is recognized as one of the major limitations in catalysis. ${ }^{5}$ Therefore, supported metal nanoparticles were developed to improve the stability and catalytic performance. ${ }^{6-9}$ Until now, various methods including impregnation, ion exchange, deposition-precipitation and atomic layer deposition have been used to control metal-support interactions, and to optimize catalytic activity. ${ }^{10}$ Most of these methods suffer from involved procedures that result in relatively low loading of metal nanoparticles
$(<20 \%)$. Therefore, there is a strong demand for a new synthetic strategy that allows high loadings of various metal nanoparticles, uniformly dispersed and spatially separated on solid supports.

To date, various supports such as polymers, ${ }^{11}$ oxides ${ }^{12-14}$ (for example, $\mathrm{SiO}_{2}, \mathrm{Al}_{2} \mathrm{O}_{3}, \mathrm{TiO}_{2}$ and $\mathrm{CeO}_{2}$ ), metal organic frameworks ${ }^{15}$ and carbon materials ${ }^{16-19}$ have been employed for loading metal nanoparticles. Among them, carbon nanospheres have been considered as an ideal platform because of their good thermal stability under inert atmosphere, intrinsic hydrophobic nature, presence of vast functional groups facilitating metal loading, and spherical morphology ensuring fast mass transfer and minimal viscous effects. ${ }^{20-22}$ Several attempts have been reported to decorate nanoporous carbonaceous spheres with metal nanoparticles. The most popular method is to reduce metal ions on the as-prepared carbon nanoparticles via thermal or chemical reduction. For example, Schüth and co-workers tested a method involving thermal reduction of metal ions confined in carbon nanopores to obtain noble-metal nanoparticles encapsulated in hollow carbon nanospheres. ${ }^{23-25}$ However, no matter

\footnotetext{
${ }^{1}$ Australian Institute for Bioengineering and Nanotechnology, The University of Queensland, Brisbane, QLD, Australia; ${ }^{2}$ Laboratory for Catalysis Engineering, School of Chemical and Biomolecular Engineering, The University of Sydney, Sydney, NSW, Australia; ${ }^{3}$ Unité de Catalyse et Chimie du Solide (UCCS), CNRS UMR 8181, Université Lille, Lille, France; ${ }^{4}$ Department of Chemistry, Kent State University, Kent, OH, USA and ${ }^{5}$ Department of Chemical Engineering, Curtin University, Perth, WA, Australia

6These authors contributed equally to this work.

Dedicated to Professor Alfons Baiker on the occasion of his 70th birthday.

Correspondence: Associate Professsor Dr J Huang, Laboratory for Catalysis Engineering, School of Chemical and Biomolecular Engineering, The University of Sydney, Sydney, NSW, 2006, Australia.

E-mail: jun.huang@sydney.edu.au

or Professsor Dr MJ Monteiro, Australian Institute for Bioengineering and Nanotechnology, The University of Queensland, Brisbane, QLD 4072, Australia.

E-mail: m.monteiro@uq.edu.au

or Dr J Liu, Department of Chemical Engineering, Curtin University, GPO Box U1987, Perth, WA 6845, Australia.

E-mail: jian.liu@curtin.edu.au

Received 16 April 2015; revised 29 October 2015; accepted 4 November 2015
} 
how the as-synthesized metal nanocrystals were prepared, by surfactant-assisted, microemulsion or polyol reaction methods, ${ }^{26-28}$ the ligands stabilizing the as-synthesized metal nanocrystals weakened the interactions between metal active sites and reactants, resulting in a decreased catalytic activity. Furthermore, because of the lack of suitable functional groups in the inert pre-synthesized carbon spheres, the post-synthetically loaded metal nanoparticles were non-uniform and randomly distributed onto the carbon spheres. Therefore, the development of a general and facile method for loading high amounts of small and uniform metal nanocrystals that are homogeneously distributed on the surface of carbon spheres has so far been elusive.

Here, we report a general coordination chemistry-based method for the in situ confinement of various metal nanocrystals such as $\mathrm{Au}$, $\mathrm{Pt}, \mathrm{Rh}, \mathrm{Ru}, \mathrm{Ag}, \mathrm{Pd}$, Ir and their bimetallic alloy counterparts within nitrogen doped carbon nanospheres $(\mathrm{N}-\mathrm{CN})$ with mesoporous (N-MCN), hollow (N-HMCN) and yolk-shell structures (N-MCN@HMCN), respectively. The catalytic activity of the resulting $\mathrm{Au}$ and $\mathrm{Pt}$ nanoparticles loaded into carbon nanospheres was evaluated in a hydrogenation reaction of aromatic ketones, and tested for their chemoselectivity even for thermodynamically disfavored unsaturated alcohols and toluene in water. The unique features of these carbon supported metal nanoparticles are (i) high uniformity and monodispersity of metal nanoparticles over carbon nanospheres; (ii) aggregate-free loading even at higher amounts ( $>40 \%)$; and (iii) possibility for incorporating diverse metal species and their bimetallic counterparts into carbon spheres.

\section{MATERIALS AND METHODS}

\section{Chemicals and reagents}

Chemicals: Hexadecyltrimethylammonium bromide (99\%), Pluronic F127, tetraethylorthosilicate (99\%), resorcinol (99\%), 3-aminophenol (98\%), ammonium hydroxide solution (25\%), formaldehyde aqueous solution (37\%) and all metal salts $\left(\mathrm{K}_{2} \mathrm{PtCl}_{6}, \mathrm{AuCl}_{3}, \mathrm{RhCl}_{3}, \mathrm{RuCl}_{3}, \mathrm{AgNO}_{3}, \mathrm{PdNO}_{3}\right.$ and $\left.\mathrm{IrCl}_{3}\right)$ were purchased from Sigma-Aldrich company (Castle Hill, NSW, Australia). Water was purified by a Milli Q system and had an electrical resistance of $18 \mathrm{M} \Omega \bullet \mathrm{cm}$.

\section{N-MCN@Metal nanospheres (route A)}

In all, $100 \mathrm{mg}$ of as-synthesized mesoporous aminophenol formaldehyde (APF) nanospheres was prepared as reported previously ${ }^{29}$ and mixed with $10 \mathrm{ml}$ of different concentration metal salt solutions $\left(\mathrm{K}_{2} \mathrm{PtCl}_{6}, \mathrm{AuCl}_{3}, \mathrm{RhCl}_{3}, \mathrm{RuCl}_{3}\right.$, $\mathrm{AgNO}_{3}, \mathrm{PdNO}_{3}$ and $\mathrm{IrCl}_{3}$ ) under ultrasonication for $10 \mathrm{~min}$ and kept under stirring for $24 \mathrm{~h}$. After that, the product APF@metal nanospheres were obtained by centrifugation and dried overnight at room temperature in a vacuum oven. To obtain N-MCN@metal nanospheres, APF@metal nanospheres were pyrolysed under $\left(5 \% \mathrm{H}_{2}\right) \mathrm{N}_{2}$ flow in a tube furnace using a heating rate of $1{ }^{\circ} \mathrm{C}$ per min up to $350^{\circ} \mathrm{C}$, dwell for $2 \mathrm{~h}$, and resuming heating rate at $1{ }^{\circ} \mathrm{C}$ per min up to $700{ }^{\circ} \mathrm{C}$ and dwell for $4 \mathrm{~h}$. According to the concentration of metal salts, the final products were denoted as N-MCN@Metal- $x$, where $x$ represents the initial concentration of metal salts solution in mM (see Supplementary Table S1).

\section{N-HMCN@Metal rattle type nanospheres (route B)}

In all, $100 \mathrm{mg}$ of as-synthesized mesoporous APF nanospheres was prepared as reported before ${ }^{29}$ and mixed with $10 \mathrm{ml}$ of $2 \mathrm{~mm}$ metal salt solution $\left(\mathrm{K}_{2} \mathrm{PtCl}_{6}\right.$, $\mathrm{AuCl}_{3}, \mathrm{RhCl}_{3}$ and $\mathrm{RuCl}_{3}$ ) under ultrasonication for $10 \mathrm{~min}$ and kept under stirring for $24 \mathrm{~h}$. Then, above solution was mixed with $75 \mathrm{mg}$ of hexadecyltrimethylammonium bromide, $15 \mathrm{ml}$ of water, $15 \mathrm{ml}$ of ethanol and $250 \mu \mathrm{l}$ of ammonium solution. After $30 \mathrm{~min}, 200 \mu \mathrm{l}$ of tetraethylorthosilicate was added and kept under stirring for another 6 h. After that, the product APF@metal@_ silica nanospheres were obtained by centrifugation and dried overnight at room temperature in a vacuum oven. To obtain N-HMCN@metal@silica nanospheres, APF@metal@silica nanospheres were calcinated under $\left(5 \% \mathrm{H}_{2}\right) \mathrm{N}_{2}$ flow in a tube furnace using a heating rate of $1{ }^{\circ} \mathrm{C}$ per min up to $350^{\circ} \mathrm{C}$, dwell for $2 \mathrm{~h}$, and resuming heating rate at $1{ }^{\circ} \mathrm{C}$ per min up to $700^{\circ} \mathrm{C}$ and dwell for
$4 \mathrm{~h}$. The resulting N-HMCN@metal nanospheres were obtained after removal of silica with HF solution (see Supplementary Table S1).

\section{N-MCN@M1@N-MCN@M2 yolk-shell nanospheres (route C)}

First, metal 1-APFs@silica core-shell nanoparticles were dispersed in $0.2 \mathrm{M} \mathrm{HCl}$ methanol solution at $60^{\circ} \mathrm{C}$ during $6 \mathrm{~h}$; this process was repeated twice to remove the remaining surfactant. Then, $40 \mathrm{mg}$ of the surfactant-free metal 1-APFs@silica core-shell nanoparticles was dispersed in the mixture of $25 \mathrm{ml}$ of water, $8.2 \mathrm{ml}$ of ethanol, $15 \mathrm{mg}$ of hexadecyltrimethylammonium bromide and $169 \mu \mathrm{l}$ of ammonium solution. After $30 \mathrm{~min}, 50 \mathrm{mg}$ of aminophenol and $70 \mu \mathrm{l}$ of formaldehyde were added respectively before leaving reaction for another $24 \mathrm{~h}$. Finally, the mixture was transferred to autoclave for hydrothermal treatment at $100^{\circ} \mathrm{C}$ for another $24 \mathrm{~h}$. The resulting metal 1-APFs@silica@APFs sample was obtained by washing with water and ethanol for three times. To prepare metal 1-APFs@silica@M2-APF nanoparticles, the above metal 1-APFs@silica@APF nanoparticles were dispersed in metal 2 salt aqueous solution (10 mM) for another 12 h. Next, these metal 1-APFs@silica@M2-APF nanoparticles were centrifuged and dried in a vacuum desiccator for $10 \mathrm{~h}$. To obtain (N-MCN@M1)@SiO $@(\mathrm{~N}-\mathrm{MCN} @ \mathrm{M} 2)$ nanoparticles, the above metal 1-APFs@silica@M2-APF nanoparticles were subjected to pyrolysis treatment under $\left(5 \% \mathrm{H}_{2}\right) \mathrm{N}_{2}$ flow in a tube furnace using a heating rate of $1{ }^{\circ} \mathrm{C} / \mathrm{min}$ up to $350{ }^{\circ} \mathrm{C}$, dwell for $2 \mathrm{~h}$, and resuming heating rate at $1{ }^{\circ} \mathrm{C} / \mathrm{min}$ up to $700{ }^{\circ} \mathrm{C}$ and $\mathrm{dwell}$ for $4 \mathrm{~h}$. Finally, the sample was cooled down to room temperature before taking it out from tube furnace. The resulting (N-MCN@M1)@(N-MCN@M2) yolk-shell structured nanospheres were obtained after removal of silica with HF solution (see Supplementary Table S1).

\section{Characterization}

Transmission electron microscopy (TEM) measurements were conducted on a JEM-2100F microscope (JEOL, Tokyo, Japan) operated at $200 \mathrm{kV}$. The samples for TEM measurement were suspended in ethanol and supported onto a holey carbon film on a $\mathrm{Cu}$ grid. Scanning electron microscopy was taken with a JEOL-7800F field emission electron microscopy. The $\mathrm{N}_{2}$-sorption experiments were performed at $-196^{\circ} \mathrm{C}$ on a Micromeritics Tristar 3000 (Atlanta, GA, USA) system with micropore analysis. Before the measurement, the samples were out-gassed at $120^{\circ} \mathrm{C}$ for at least $6 \mathrm{~h}$. The Brunauer-Emmett-Teller specific surface areas were calculated using adsorption data at a relative pressure range of $P / P_{0}=0.05-0.25$. The total pore volumes were estimated from the amount of nitrogen adsorbed at a relative pressure $\left(P / P_{0}\right)$ of 0.99 . X-ray photoelectron spectroscopy (XPS) data were acquired using a Kratos Axis ULTRA X-ray Photoelectron Spectrometer (Kratos Analytical Ltd, Manchester, UK), their atomic concentrations were calculated using the CasaXPS version 2.3.14 software (Casa Software Ltd., Cheshire, UK) and a Shirley baseline with Kratos library Relative Sensitivity Factors. Peak fitting of the high-resolution data was also carried out using the CasaXPS software.

\section{Hydrogenation evaluation}

The catalysts were pretreated under $\mathrm{H}_{2}$ with a flow rate of $40-50 \mathrm{ml} \mathrm{min}^{-1}$ for $1 \mathrm{~h}$ at $400^{\circ} \mathrm{C}$. Hydrogenation of benzaldehyde was carried out in an aqueous phase in a stainless-steel autoclave with a magnetic stirrer. The autoclave was filled with the reaction mixture consisting of a substrate $(0.05 \mathrm{M}$, benzaldehyde), water $(6 \mathrm{ml})$ and catalyst $(4-30 \mathrm{mg}$, corresponding to $1.7 \mathrm{mg}$ of metal, and the the molar ratio of 35), unless specified otherwise. The autoclave was pressurized with hydrogen $(0.3 \mathrm{MPa})$ and heated to the required reaction temperature (room temperature or $80^{\circ} \mathrm{C}$ ).

\section{RESULTS}

The fabrication of metal nanoparticle-loaded carbon nanospheres As illustrated in Figure 1, first, the mesoporous APF polymer resin nanospheres with uniformly distributed amino groups were prepared via a dual surfactant templating method according to our previous report. ${ }^{29}$ Second, nitrogen-doped mesoporous carbon nanospheres decorated with monodispersed metal nanocrystals (N-MCN@M) were formed via coordination-type interactions between metal ions and amine groups, followed by thermal reduction of metal ions to form 


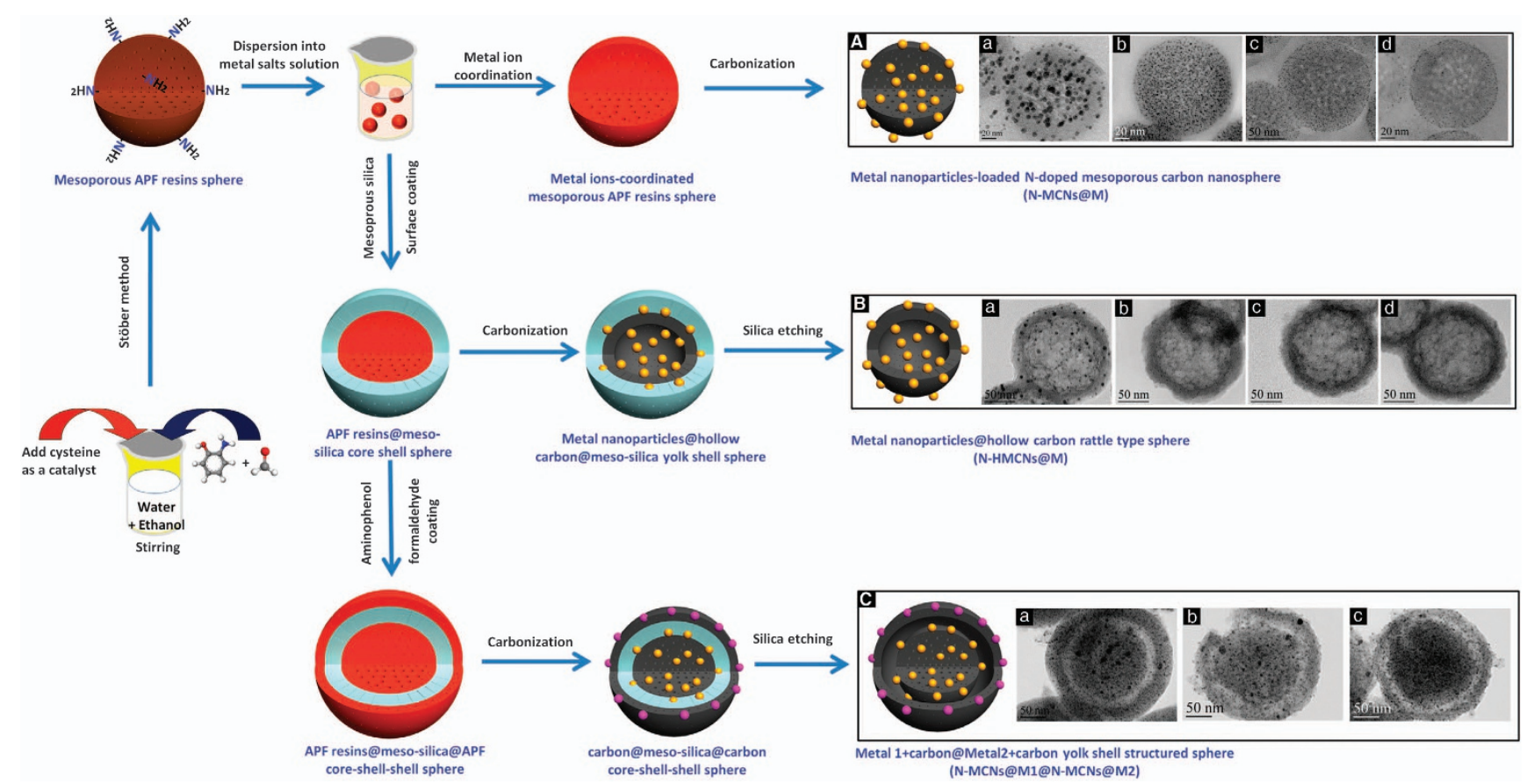

Figure 1 Schematic illustration of three general routes for the formation of various types of nanospheres using aminophenol-formaldehyde (APF) resin: (route A) N-MCN@M with cross-section TEM images showing (a) N-MCN@Au, (b) N-MCN@Pt, (c) N-MCN@Rh and (d) N-MCN@Ru; (route B) N-HMCN@M with TEM images showing (a) N-HMCN@Au, (b) N-HMCN@Pt, (c) N-HMCN@Rh and (d) N-HMCN@Ru; (route C) yolk-shell (N-MCN@HMCN) spheres with noble-metal nanoparticles, being as a combination of N-MCN core and N-HMCN shell with the corresponding TEM images for (a) (N-MCN@Au)@(HMCN@Pt), (b) (N-MCN@Au)@(HMCN@Rh) and (c) (N-MCN@Au)@(HMCN@Rh).

the metal nanoparticles (Figure 1, route A). The cross-section TEM images in Figure $1 \mathrm{~A}(\mathrm{a}-\mathrm{d})$ show monodispersed metal nanoparticles $(\mathrm{Au}, \mathrm{Pt}, \mathrm{Rh}$ and $\mathrm{Ru})$ onto N-MCNs. We believe that the pore channels in mesoporous APFs resin spheres have a dominant role as nanocontainers to confine the growth of metal nanocrystals during thermal reduction. This resulted in very small and uniform metal nanoparticle sizes even after high temperature carbonization. For N-MCN@Au, the mean $\mathrm{Au}$ particle size was estimated to be $6.1 \pm 3.0 \mathrm{~nm}$. In the case of $\mathrm{N}-\mathrm{MCN}$ decorated with other metal nanoparticles, the mean particle size of $\mathrm{Pt}, \mathrm{Rh}$ and $\mathrm{Ru}$ was estimated to be $2.5 \pm 1.5,2.2 \pm 0.8$ and $2.5 \pm 1.0 \mathrm{~nm}$, respectively. The distribution of homogeneous metal nanoparticles was confirmed by the energy-dispersive X-ray spectroscopy spectra (Supplementary Figure S1). The formation of metal nanoparticles was further confirmed by the XRD patterns. In the case of Au nanoparticles, which possessed larger sizes than the other metals because of faster nucleation of $\mathrm{Au}$, the XRD patterns exhibited distinct reflections at $2 \Theta$ of 44,52 and $76^{\circ}$ attributed to the 111, 200 and 220 peaks of the face-centred cubic (fcc) Au nanocrystals (Supplementary Figure S2). The lattice distance corresponding to (111) diffraction peak was $2.35 \AA$, which is in a good agreement with the Au lattice fringe estimated by analysis of the high resolution transmission electron microscopy (HRTEM) images (Supplementary Figure S3). The Au particle size estimated from the (111) peak using the Scherer formula was around $6.1 \mathrm{~nm}$, which also agrees well with the particle size observed by TEM $(6.0 \mathrm{~nm})$. The XPS spectra and electron energy loss spectroscopy mapping data show that the metal decorated $\mathrm{N}-\mathrm{MCN}$ s consisted of carbon, nitrogen, oxygen and metal species corresponding to the $\mathrm{Au}, \mathrm{Pt}, \mathrm{Rh}$ and $\mathrm{Ru}$ metal ions used in the synthesis (Supplementary Figures S4 and S5). To demonstrate the generality of the synthetic method, Supplementary Figure S6 shows the TEM, XPS and XRD characterization results for N-MCN decorated with $\mathrm{Ag}$, Pd and Ir nanoparticles. The dominant interaction between carbon precursor and metals is coordination, in which aminophenol can provide amine functional groups for coordination of metal ions. Moreover, the thermal reduction process involving consumption of carbon and reduction of metal in nanoparticles is favorable for formation of uniformly dispersed metal nanoparticles embedded in carbon spheres. ${ }^{30,31}$ It is believed that the concave curvature on the carbon sphere surface, which was generated during the thermal reduction process, allows for homogeneous formation of metal nanoparticles in the N-doped carbon spheres.

We further demonstrate that metal nanoparticles can be confined in the interior of $\mathrm{N}$-doped hollow mesoporous carbon nanospheres to form the rattle-type nanoparticles with metal cores and hollow $\mathrm{N}$-doped carbon shells (N-HMCN@M) (Figure 1, route B, and Supplementary Figure S7). Because the noble-metal nanoparticles were obtained by thermal reduction of coordinated metal ions, this synthesis route can be easily applicable to other metal species such as $\mathrm{Au}, \mathrm{Pt}, \mathrm{Rh}$ and $\mathrm{Ru}$ (Figure $1 \mathrm{~B}(\mathrm{a}-\mathrm{d})$ ). The entrapped metal ions inside carbon spheres can be reduced to metal nanoparticles at high temperature and confined in these spheres (Supplementary Figure S8). The shell thickness of the metal confined N-HMCNs was assessed to be around $25 \mathrm{~nm}$; and the mean particle sizes of $\mathrm{Au}, \mathrm{Pt}, \mathrm{Rh}$ and $\mathrm{Ru}$ were estimated to be 5.4, 2.0, 1.8 and $2.2 \mathrm{~nm}$, respectively (Figure $1 \mathrm{~B}(\mathrm{a}-\mathrm{d})$ and Supplementary Figure S8). The shells of these nanoparticles were mesoporous as evidenced by the type IV shape of adsorption-desorption isotherms having a H4 hysteresis loop.

The layer-by-layer method was used to selectively deposit bimetallic nanoparticles on the yolks and shells of $\mathrm{N}$-doped yolk-shell carbon nanospheres (N-MCN@HMCN) (Figure 1, route C). TEM images Figure $1 \mathrm{C}(\mathrm{a}-\mathrm{c})$ indicate that the metal catalyst nanoparticles were uniformly distributed in/on the mesoporous carbon cores and microporous shells. The scanning electron microscopy images (Supplementary Figure S9) show broken yolk-shell spheres 

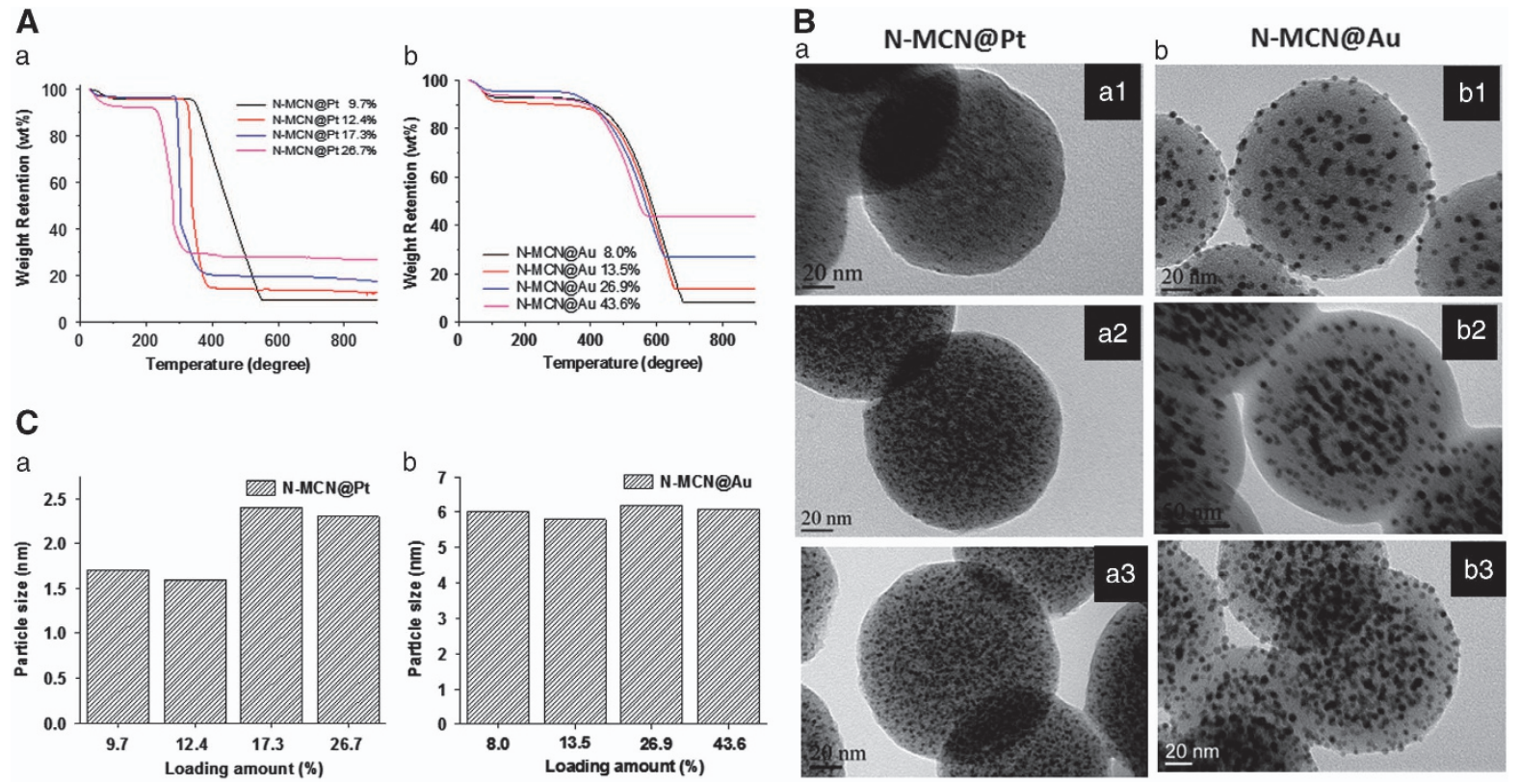

Figure 2 (A) The TG profiles of (a) N-MCN@Pt and (b) N-MCN@Au nanospheres calcined in air. (B) TEM images of (a) N-MCN@Pt with different loadings of Pt nanoparticles (wt\%) (a1) 12.4\%, (a2) 17.3\% and (a3) 26.7\%; (b) N-MCN@Au with different loadings of Au nanoparticles (wt\%) (b1) 13.5\%, (b2) 26.9\% and (b3) $43.6 \%$. (C) The average size of metal nanoparticles vs loading (wt\%) of these nanoparticles in (a) N-MCN@Pt and (b) N-MCN@Au spheres.

having mesoporous carbon cores decorated with metal nanoparticles. $\mathrm{N}_{2}$ adsorption isotherms with $\mathrm{H} 4$ hysteresis loop and the shape being a combination of types I and IV (Supplementary Figure S10a), and the corresponding pore size distributions (Supplementary Figure S10b) indicate that each yolk-shell structure consists of an empty space between the microporous carbon core and mesoporous carbon shell. The aforementioned three different synthesis routes were used to design and synthesize a series of catalysts with different metal nanoparticles, morphologies and structures as confirmed by their detailed characterization.

\section{Varying the loading of metal nanoparticles}

Furthermore, the loading of metal nanoparticles can be conveniently controlled by tuning the initial concentration of the metal salts used. The TG profiles shown in Figure 2A indicate that the Pt loading can be tailored from 9.7 to $26.7 \%$ in the N-MCN@Pt samples, while the loading of $\mathrm{Au}$ nanoparticles can be altered from 8 to $44 \%$ in the N-MCN@Au samples. Interestingly, the carbon spheres with high loading of Pt nanoparticles showed better catalytic oxidation performance as evidenced by lower carbon oxidation temperature (that is, 220 instead of $340{ }^{\circ} \mathrm{C}$ ). The TEM images of N-MCN@M (Figure 2B) indicate that the amount of metal nanoparticles in each carbon nanosphere increases with increasing concentration of metal salt. Surprisingly, even at the metal loading as high as $44 \%$ these metal nanoparticles are still uniformly dispersed and spatially separated on the carbon support (Figure 2B and Supplementary Figures S11-S15). As shown in Supplementary Figure S13, the Pt nanoparticle size increased gradually from 1.5 to $2.4 \mathrm{~nm}$ with increasing Pt loading from 12.4 to $26.7 \mathrm{wt} \%$, whereas the Au nanoparticle size does not change with increasing Au loading from 13.5 to $43.6 \mathrm{wt} \%$. In addition, the mesoporous structure of carbon spheres was quite well preserved at the concentration of metal salt smaller than $10 \mathrm{~mm}$ (Supplementary Figure S16). Figure 2C shows the relationship between the metal particle size and the corresponding metal loading in the N-MCN@Pt and N-MCN@Au samples. As can be seen, the size of Pt particles tends to increase with increasing metal loading; however, the size of $\mathrm{Au}$ particles is independent of the loading of $\mathrm{Au}$. A possible explanation is the presence of $-\mathrm{NH}_{2}$ groups in mesoporous APFs resin spheres, which interact strongly with $\mathrm{Au}$ species and limit the growth of $\mathrm{Au}$ nanoparticles in the pore channels.

The above synthesis route can be further extended to the fabrication of mesoporous carbon spheres with bimetallic nanoparticles, AuPt, AuRh and PtRh (N-MCN@BM). The scanning electron microscopy (Supplementary Figure S17a-c) and TEM (Supplementary Figure S17d-f) images show bimetallic nanoparticles homogeneously deposited on the surface and inside pore channels without visible aggregation. The cross-section TEM images and energy-dispersive X-ray spectroscopy spectra (Supplementary Figure S18) indicate that the binding capacity of bimetallic nanoparticles in $\mathrm{N}-\mathrm{MCN}$ followed the sequence $\mathrm{Pt}>\mathrm{Au}>\mathrm{Rh}$, which is in a good agreement with their surface loading estimated on the basis of the XPS data (Supplementary Figure S19). Nitrogen adsorption isotherms obtained for N-MCN@bimetallic nanoparticles are shown in Supplementary Figure S20; they resemble a combination of types I and IV isotherms, indicating the presence of micropores and mesopores. In addition, the bimetallic nanoparticles were found to be concentrated in the cores to form rattle type bimetallic cores confined in hollow mesoporous carbon nanospheres with high surface areas, denoted as N-HMCN@BM, obtained via the silica-assisted synthesis (Figure 3 and Supplementary Figure S21). Thus, this method represents a facile strategy to synthesize hollow carbon spheres with confined bimetals, which would have potential applications in many conventional catalytic processes.

\section{Catalytic performance}

Catalytic performance of these catalysts was evaluated for hydrogenation of benzaldehyde in water using a high-pressure reactor. Benzaldehyde is a renewable chemical compound of bio-oil obtained by partial pyrolysis of biomass. ${ }^{32,33}$ The catalytic hydrogenation of benzaldehyde is a model reaction for hydrogenation of aromatic aldehydes leading to alcohol (benzyl alcohol) or hydrocarbons 

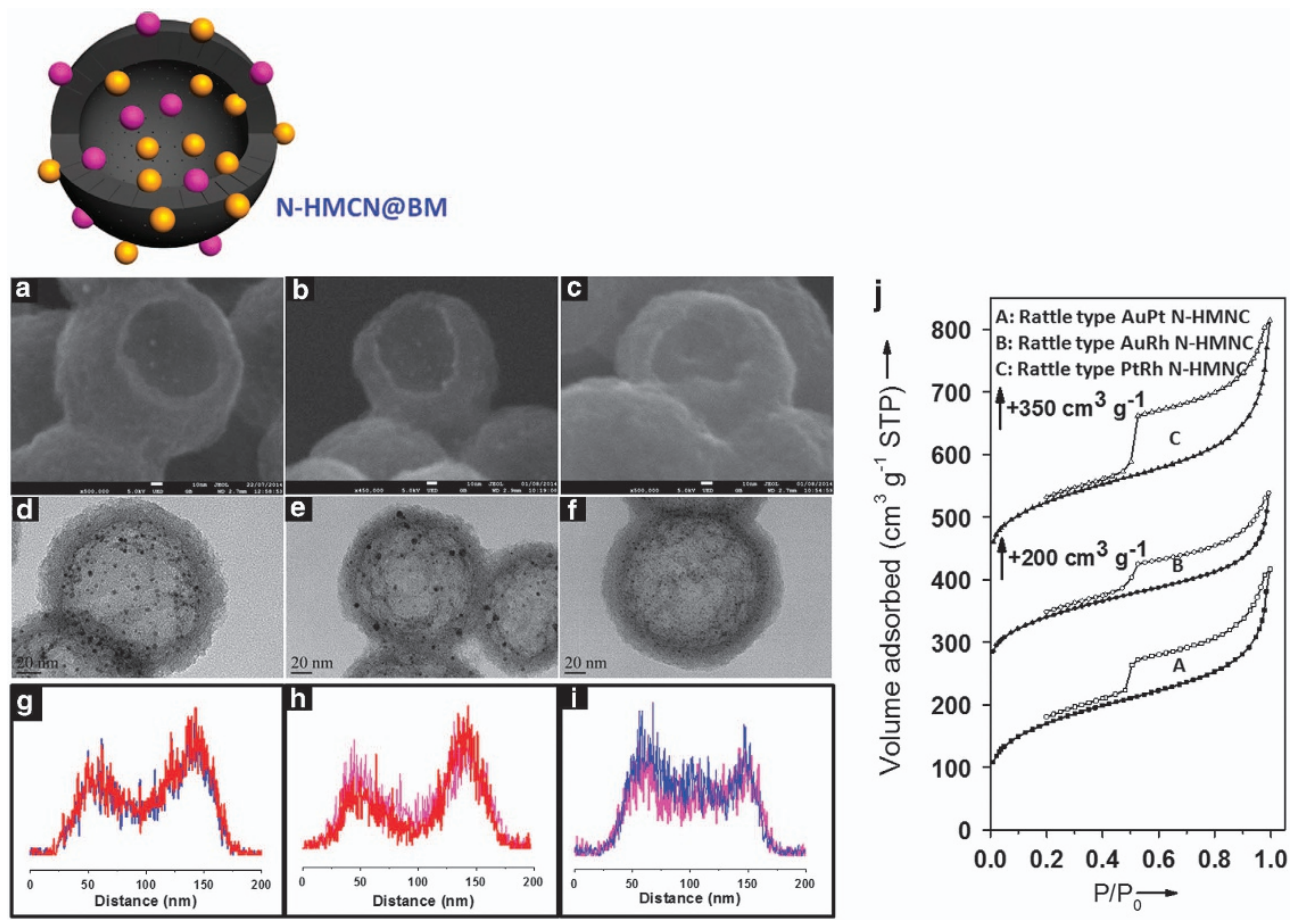

Figure 3 (a-c) HRSEM images, (d-f) high resolution transmission electron microscopy images, (g-i) EDS spectra and (j) N2 adsorption desorption isotherms of (a, d and $\mathbf{g}$ ), N-HMCN@AuPt, (b, e and $\mathbf{h}$ ), N-HMCN@AuRh, (c, f and i) and N-HMCN@PtRh, respectively.

(toluene or benzene). In general, hydrogenation of benzaldehyde can generate benzyl alcohol, toluene, benzene and other byproducts because of the competitive hydrogenation between the $\mathrm{C}=\mathrm{O}$ and phenyl groups (see Supplementary Figure S22a). ${ }^{34}$ However, the selectivity toward toluene is of primary importance, as this aromatic hydrocarbon is widely used as (i) an industrial feedstock (precursor to other chemicals), (ii) a solvent (for paints, printing ink, adhesives and disinfectants) and (iii) an additive in gasoline fuels (octane booster) used in internal combustion engines. ${ }^{32-35}$ Our carbon spheres with highly dispersed $\mathrm{Pt}$ and Au catalysts were highly active and chemoselective for hydrogenation toward the $\mathrm{C}=\mathrm{O}$ group at the room temperature (Supplementary Table S2). In the case of Au catalysts, toluene was the only product at $100 \%$ conversion of benzaldehyde (Figure 4). The results are consistent with earlier reports on hydrogenation over gold nanoparticles with sizes of about $5-8 \mathrm{~nm} .{ }^{34-36}$ This study shows that the catalytic activity of $\mathrm{Pt}$, which has been considered as the best hydrogenation catalyst, is much lower (turn over frequencies, TOF from 12 to $14 \mathrm{~h}^{-1}$ ) than that of the corresponding Au catalysts (TOF from 30 to $45 \mathrm{~h}^{-1}$ ) for hydrogenation in water.

\section{DISCUSSION}

By increasing the reaction temperature, one can enhance the hydrogenation rate for both $\mathrm{Pt}$ and $\mathrm{Au}$ catalysts as confirmed by the enlarged TOF values at $80^{\circ} \mathrm{C}$ in comparison with those at room temperature (Supplementary Table S2). However, at higher temperature the catalytic activity of $\mathrm{Pt}$ and $\mathrm{Au}$ is opposite to that observed at room temperature; the TOF values $102-478 \mathrm{~h}^{-1}$ for Pt catalysts are much higher than those obtained for Au catalysts $\left(45-79 \mathrm{~h}^{-1}\right)$. Up to $80^{\circ} \mathrm{C}$ (Supplementary Figure S23), the conversion of benzaldehyde was greater than $70 \%$ in just $5 \mathrm{~min}$ and reached $100 \%$ in $60 \mathrm{~min}$ over N-MCN@Pt 9.6\% and N-MCN@Pt 26.7\%; these values are much higher than those obtained on $\mathrm{Au}$ catalysts. The size of metal nanoparticles has a significant effect on their catalytic activity; ${ }^{37}$ metal catalysts with small particle sizes usually show better catalytic activity. ${ }^{38}$ However, if the particle size is too small, then the catalytic activity can be also reduced. ${ }^{39}$ According to our results, N-MCN@Pt $26.7 \%$ particles with sizes of $2.3 \mathrm{~nm}$ showed higher activity toward hydrogenation of benzaldehyde and higher selectivity to toluene (TOF: $478 \mathrm{~h}^{-1}$; selectivity: 91\%) in comparison with N-MCN@Pt $9.6 \%$ with a particle size of $1.7 \mathrm{~nm}$ (TOF: $396 \mathrm{~h}^{-1}$; selectivity: $87 \%$ ). Similarly, Prashar et al. ${ }^{37}$ reported that $\mathrm{Pt} / \mathrm{SiO}_{2}$ with relatively larger $\mathrm{Pt}$ particles with sizes of $8 \mathrm{~nm}$ displayed better catalytic performance (selectivity: $79 \%$ at $75 \%$ conversion) toward hydrogenation of cinnamaldehyde to cinnamyl than $\mathrm{Pt} / \mathrm{SiO}_{2}$ with a mean $\mathrm{Pt}$ size of $1.8 \mathrm{~nm}$ (selectivity: $57 \%$ at $75 \%$ conversion). Also, another reports ${ }^{34-36}$ demonstrated that $\mathrm{Au} / \mathrm{TiO}_{2}$ with a mean $\mathrm{Au}$ particle size of $5.3 \mathrm{~nm}$ exhibited higher activity $\left(513 \mu \mathrm{mol} \mathrm{g}^{-1} \mathrm{Au} \mathrm{s}^{-1}\right)$ than that of an analogous catalyst with a mean Au size of $2.0 \mathrm{~nm}$ $\left(400 \mu \mathrm{mol} \mathrm{g}^{-1} \mathrm{Au} \mathrm{s}^{-1}\right)$ during catalytic hydrogenation of acrolein to allyl alcohol. In this research, N-MCN@Au catalysts with 8.0 and $26.8 \%$ of $\mathrm{Au}$ and similar mean Au particle sizes of $6 \mathrm{~nm}$ preformed slightly different (TOF: 52 and $45 \mathrm{~h}^{-1}$, respectively), which may cause some difference in the internal diffusion of reactant/product inside both catalysts. Namely, the higher pore volume $\left(0.26 \mathrm{~cm}^{3} \mathrm{~g}^{-1}\right)$ of the N-MCN@Au 8.0\% catalyst may offer better internal diffusion as compared with N-MCN@Au 26.8\% $\left(0.17 \mathrm{~cm}^{3} \mathrm{~g}^{-1}\right)$. The different performance of $\mathrm{Pt}$ and $\mathrm{Au}$ catalysts at different temperatures may be influenced by the ad/desorption rates on the surface. The Pt sites could strongly adsorb reactants and products, which reduce the desorption rate of the catalytic cycle on the Pt surface and produce a relatively low TOF. ${ }^{38,40}$ An increase in the reaction temperature would promote a faster ad/desorption of products on/from the Pt surface. Generally, $\mathrm{H}_{2}$ dissociation is considered as a key step in hydrogenation reactions. The desorption activation energy for $\mathrm{H}_{2}$ over $\mathrm{Au}(111)$ is estimated to be $\sim 28 \mathrm{~kJ} \mathrm{~mol}^{-1}(0.29 \mathrm{eV})$, while over $\mathrm{Pt}(111)$ is about $0.74-0.87$ $\mathrm{eV}^{40} \mathrm{~A}$ low desorption activation energy of $\mathrm{Au}(111)$ suggests a weak 

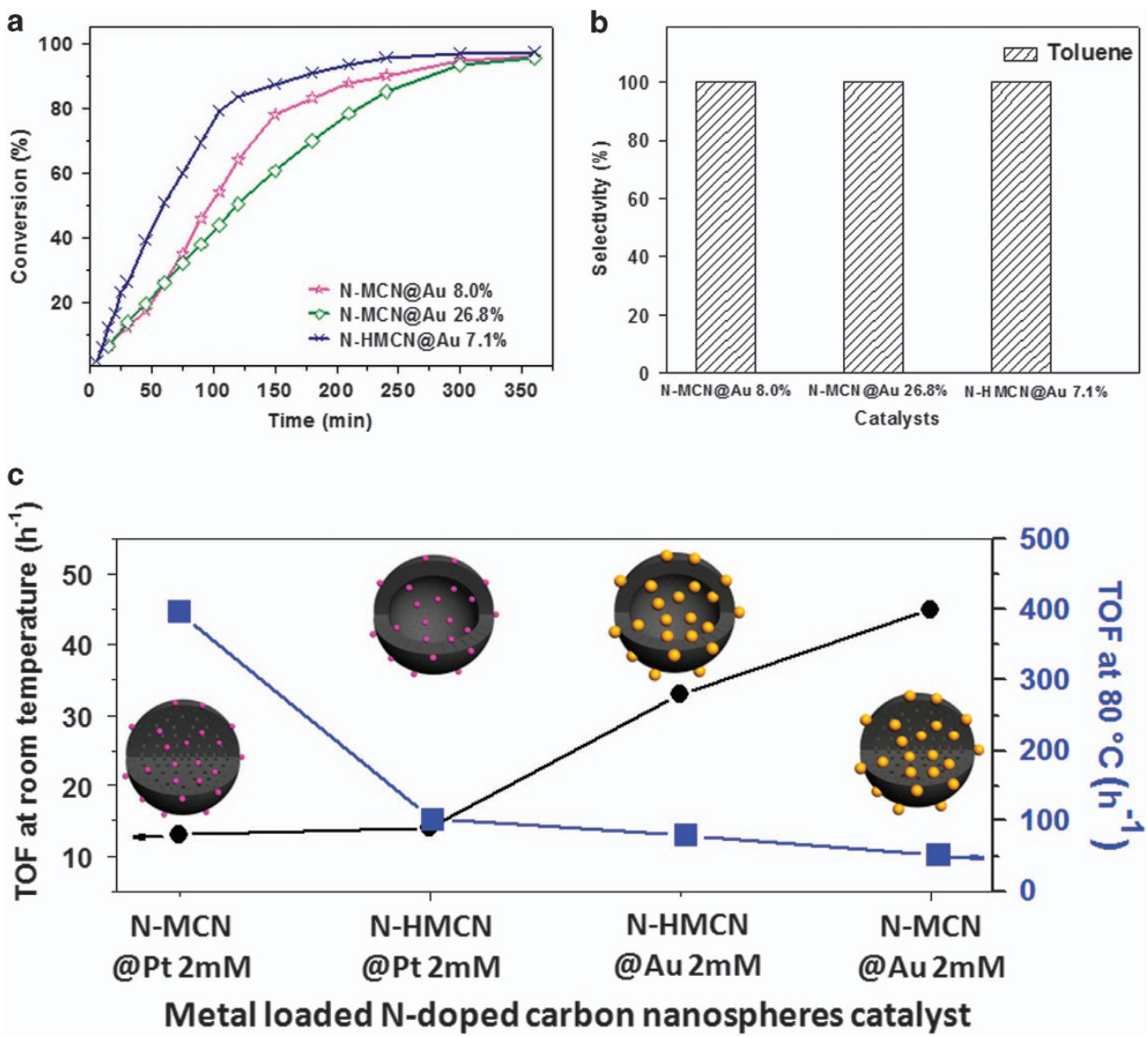

Figure 4 (a) The conversion of benzaldehyde to benzyl alcohol and toluene catalyzed by N-MCN@Au with different Au loadings at $80^{\circ} \mathrm{C}$; (b) the corresponding selectivity of benzaldehyde to benzyl alcohol and toluene; and (c) catalyst profile vs turn over frequencies (TOF) at different reaction temperatures.

interaction between atomic $\mathrm{H}$ and the $\mathrm{Au}(111)$ surface. ${ }^{40}$ Therefore, more active species are possible on the gold surface because single $\mathrm{H}$ atoms interact very weakly with $\mathrm{Au}$ as compared to other metallic surfaces.

The strongly enhanced reaction activity/conversion on the prepared catalysts at $80^{\circ} \mathrm{C}$ (for example, in the case of N-MCN@Pt 26.7\%: TOF is 40 times greater than that at room temperature) does not change the chemoselectivity of hydrogenation. Even for prolonged reaction times after $100 \%$ benzaldehyde conversion there was no evidence for hydrogenation of aromatic rings or loss of $\mathrm{C}=\mathrm{O}$ (benzene production was not detected) at $80^{\circ} \mathrm{C}$. Hydrogenation of benzaldehyde generated only two products, benzyl alcohol and toluene as shown in Supplementary Figure S23. The possibility of reusing the catalysts was also tested as shown in Supplementary Figures S24 and S25; the conversion of benzaldehyde on all the regenerated N-doped carbon supported $\mathrm{Au}$ and $\mathrm{Pt}$ catalysts was compared with that on the fresh catalysts with $100 \%$ selectivity toward carbonyl group. In addition, no leaching and sintering were detected on the catalysts used via hot filtration test (Supplementary Figure S26) and TEM imaging analysis (Supplementary Figure S27), respectively. Thus, the catalysts with monodispersed metal nanoparticles are super-highly chemoselective for hydrogenation reactions.

In conclusion, a general strategy was developed for the synthesis of versatile carbon nanospheres impregnated with highly monodispersed metal nanoparticles. The morphology and porosity of carbon spheres as well as the loading of metal nanoparticles can be well controlled.
For the first time, we demonstrated that carbon spheres could be impregnated with uniformly dispersed $\mathrm{Au}$ and $\mathrm{Pt}$ nanoparticles at loadings as high as $44 \mathrm{wt} \%$. A morphology-dependent catalysis on Au nanoparticles was found for hydrogenation of benzaldehyde at room temperature, which is reflected by the highest activity (TOF $2-4$ times higher than that on the Pt particles with the same size and metal loading) and selectivity (100\% to toluene). It is expected that the carbon spheres loaded with the aforementioned metal nanoparticles and other transition metal nanoparticles will provide an ideal platform for understanding the relation between structure and catalytic activity.

\section{CONFLICT OF INTEREST}

The authors declare no conflict of interest.

\section{ACKNOWLEDGEMENTS}

This work was financially supported by the Australian Research Council (ARC) through Discovery Project program (DP1094070) and Linkage Project program (LP150101158). JL gratefully acknowledges the support of UQ Foundation Research Excellence Award, France-Australia Science Innovation Collaboration (FASIC) program Early Career Fellowships and Curtin University Pro Vic-Chancellor Awards for Research Excellence. JH acknowledges the MCR Scheme and the Cluster Scheme at the Faculty of Engineering \& IT, the University of Sydney. JL and MM designed the research. TYY synthesized the metal nanoparticle-loaded carbonaceous nanospheres. HJL and JH performed the hydrogenation experiments. TYY and JL performed characterizations. TYY, $\mathrm{JH}$ and JL co-wrote the manuscript with input from JFL, MJ and MM. 
1 Somorjai, G. A. Introduction to Surface Chemistry and Catalysis (Wiley-Interscience, 1994).

2 Guo, S., Zhang, S. \& Sun, S. Tuning nanoparticle catalysis for the oxygen reduction reaction. Angew. Chem. Int. Ed. 52, 8526-8544 (2013).

3 Xia, Y., Xiong, Y., Lim, B. \& Skrabalak, S. E. Shape-controlled synthesis of metal nanocrystals: simple chemistry meets complex physics? Angew. Chem. Int. Ed. 48 60-103 (2009).

4 Somorjai, G. A., Frei, H. \& Park, J. Y. Advancing the frontiers in nanocatalysis, biointerfaces, and renewable energy conversion by innovations of surface techniques. J. Am. Chem. Soc. 131, 16589-16605 (2009).

5 Bond, G. C. Supported metal catalysts: some unsolved problems. Chem. Soc. Rev. 20, 441-475 (1991).

6 Prieto, G., Zečević, J., Friedrich, H., de Jong, K. P. \& de Jongh, P. E. Towards stable catalysts by controlling collective properties of supported metal nanoparticles. Nat. Mater. 12, 34-39 (2013)

7 Wang, S., Zhao, Q., Wei, H., Wang, J. Q., Cho, M., Cho, H. S., Terasaki, O. \& Wan, Y. Aggregation-free gold nanoparticles in ordered mesoporous carbons: toward highly active and stable heterogeneous catalysts. J. Am. Chem. Soc. 135 11849-11860 (2013).

8 Pan, X., Fan, Z., Chen, W., Ding, Y., Luo, H. \& Bao, X. Enhanced ethanol production inside carbon-nanotube reactors containing catalytic particles. Nat. Mater. 6, 507-511 (2007).

9 Zhu, Q.-L., Li, J. \& Xu, Q. Immobilizing metal nanoparticles to metal-organic frameworks with size and location control for optimizing catalytic performance. J. Am. Chem. Soc. 135, 10210-10213 (2013).

10 Stair, P. C. Advanced synthesis for advancing heterogeneous catalysis. J. Chem. Phys. 128, 182507 (2008)

11 Richter, F. H., Meng, Y., Klasen, T., Sahraoui, L. \& Schüth, F. Structural mimicking of inorganic catalyst supports with polydivinylbenzene to improve performance in the selective aerobic oxidation of ethanol and glycerol in water. J. Catal. 308, 341-351 (2013).

12 Yamada, Y., Tsung, C. K., Huang, W., Huo, Z., Habas, S. E., Soejima, T., Aliaga, C. E., Somorjai, G. A. \& Yang, P. Nanocrystal bilayer for tandem catalysis. Nat. Chem. 3, 372-376 (2011).

13 An, K., Alayoglu, S., Musselwhite, N., Na, K. \& Somorjai, G. A. Designed catalysts from Pt nanoparticles supported on macroporous oxides for selective isomerization of n-Hexane. J. Am. Chem. Soc. 136, 6830-6833 (2014).

14 Turner, M., Golovko, V. B., Vaughan, O. P., Abdulkin, P., Berenguer-Murcia, A., Tikhov, M. S., Johnson, B. F \& Lambert, R. M. Selective oxidation with dioxygen by gold nanoparticle catalysts derived from 55-atom clusters. Nature 454, 981-983 (2008).

15 Kuo, C.-H., Tang, Y., Chou, L. Y., Sneed, B. T., Brodsky, C. N., Zhao, Z. \& Tsung, C. K. Yolk-shell nanocrystal@ZIF-8 nanostructures for gas-phase heterogeneous catalysis with selectivity control. J. Am. Chem. Soc. 134, 14345-14348 (2012).

16 Wang, S., Wang, J., Zhao, Q., Li, D., Wang, J. Q., Cho, M., Cho, H., Terasaki, O., Chen, S. \& Wan, Y. Highly active heterogeneous $3 \mathrm{~nm}$ gold nanoparticles on mesoporous carbon as catalysts for low-temperature selective oxidation and reduction in water. ACS Catal. 5, 797-802 (2014).

17 Guo, X., Fang, G., Li, G., Ma, H., Fan, H., Yu, L., Ma, C., Wu, X, Deng, D., Wei, M., Tan, D., Si, R., Zhang, S., Li, J., Sun, L., Tang, Z., Pan, X. \& Bao, X. Direct, nonoxidative conversion of methane to ethylene, aromatics, and hydrogen. Science 344, 616-619 (2014)

18 Lam, E. \& Luong, J. H. T. Carbon materials as catalyst supports and catalysts in the transformation of biomass to fuels and chemicals. ACS Catal. 4, 3393-3410 (2014).

19 Zhou, R. \& Qiao, S. Z. Silver/nitrogen-doped graphene interaction and its effect on electrocatalytic oxygen reduction. Chem. Mater. 26, 5868-5873 (2014).

20 Wickramaratne, N. P. \& Jaroniec, M. Facile synthesis of polymer and carbon spheres decorated with highly dispersed metal nanoparticles. Chem. Commun. 50, 12341-12343 (2014)

21 Liu, J., Yang, T., Wang, D. W., Lu, G. Q. M., Zhao, D. Y. \& Qiao, S. Z. A facile softtemplate synthesis of mesoporous polymeric and carbonaceous nanospheres. Nat. Commun. 4, doi:10.1038/ncomms3798 (2013)

22 Yang, T., Zhou, R., Wang, D. W., Jiang, S. P., Yamauchi, Y., Qiao, S. Z., Monteiro, M. J. \& Liu, J. Hierarchical mesoporous yolk-shell structured carbonaceous nanospheres for high performance electrochemical capacitive energy storage. Chem. Commun. 51, 2518-2521 (2015).
23 Galeano, C., Meier, J. C., Peinecke, V., Bongard, H., Katsounaros, I., Topalov, A. A., Lu, A. H., Mayrhofer, K. J., Schüth, F. Toward highly stable electrocatalysts via nanoparticle pore confinement. J. Am. Chem. Soc. 134, 20457-20465 (2012).

24 Galeano, C., Meier, J. C., Peinecke, V., Bongard, H., Katsounaros, I., Topalov, A. A., Lu, A., Mayrhofer, K. J. \& Schüth, F. Nitrogen-doped hollow carbon spheres as a support for platinum-based electrocatalysts. ACS Catal. 4, 3856-3868 (2014).

25 Wang, G.-H., Hilgert, J., Richter, F. H., Wang, F., Bongard, H. J., Spliethoff, B., Weidenthaler, C. \& Schüth, F. Platinum-cobalt bimetallic nanoparticles in hollow carbon nanospheres for hydrogenolysis of 5-hydroxymethylfurfural. Nat. Mater. 13, 293-300 (2014).

26 Guan, B., Wang, X., Xiao, Y., Liu, Y. \& Huo, Q. A versatile cooperative template-directed coating method to construct uniform microporous carbon shells for multifunctional coreshell nanocomposites. Nanoscale 5, 2469-2475 (2013).

27 Yang, T., Liu, J., Zheng, Y., Monteiro, M. J. \& Qiao, S. Z. Facile fabrication of core-shell-structured Ag@Carbon and mesoporous yolk-shell-structured Ag@Carbon@Silica by an extended Stöber method. Chem. Eur. J. 19, 6942-6945 (2013).

28 Liu, R., Mahurin, S. M., Li, C., Unocic, R. R., Idrobo, J. C., Gao, H., Pennycook, S. J. \& Dai, S. Dopamine as a carbon source: the controlled synthesis of hollow carbon spheres and yolk-structured carbon nanocomposites. Angew. Chem. Int. Ed. 50, 6799-6802 (2011)

29 Yang, T., Liu, J., Zhou, R., Chen, Z., Xu, H., Qiao, S. Z. \& Monteiro, M. J. N-doped mesoporous carbon spheres as the oxygen reduction reaction catalysts. J. Mater. Chem. A 2, 18139-18146 (2014).

30 Su, F. B., Lv, L., Lee, F. Y., Liu, T., Cooper, A. I. \& Zhao, X. S. Thermally reduced ruthenium nanoparticles as a highly active heterogeneous catalyst for hydrogenation of monaromatics. J. Am. Chem. Soc. 129, 14213-14223 (2007)

31 Liu, J., Wickramaratne, N. P., Qiao, S. Z. \& Jaroniec, M. "Molecular-based design and emerging applications of nanoporous carbon spheres". Nat. Mater. 14, 763-774 (2015).

32 Benson, T.J., Daggolu, P. R., Hernandez, R. A., Liu, S. \& White, M. G. Catalytic deoxygenation chemistry: upgrading of liquids derived from biomass processing. Adv. Catal. 56, 320-322 (2013).

33 Ausavasukhi, A., Sooknoi, T. \& Resasco, D. E. Catalytic deoxygenation of benzaldehyde over gallium-modified ZSM-5 zeolite. J. Catal. 268, 68-78 (2009).

34 Perret, N., Cárdenas-Lizana, F. \& Keane, M. A. Selective hydrogenation of benzaldehyde to benzyl alcohol over $\mathrm{Au} / \mathrm{Al}_{2} \mathrm{O}_{3}$. Catal. Commun. 16, 159-164 (2011).

$35 \mathrm{Ma}, \mathrm{Z}$. \& Dai, S. Development of novel supported gold catalysts: a materials perspective. Nano Res. 4, 3-32 (2011).

36 Schimpf, S., Lucas, M., Mohr, C., Rodemerck, U., Brückner, A., Radnik, J., Hofmeister, H. \& Claus, P. Supported gold nanoparticles: in-depth catalyst characterization and application in hydrogenation and oxidation reactions. Catal. Today $\mathbf{7 2}$, 63-78 (2002)

37 Prashar, A. K. Mayadevi, S. \& Nandini Devi, R. Effect of particle size on selective hydrogenation of cinnamaldehyde by Pt encapsulated in mesoporous silica. Catal. Commun. 28, 42-46 (2012).

38 Plomp, A. J., Vuori, H., Krause, A. O. I., Jong, K. P. D. \& Bitter, J. H. Particle size effects for carbon nanofiber supported platinum and ruthenium catalysts for the selective hydrogenation of cinnamaldehyde. Appl. Catal. A Gen. 351, 9-15 (2008).

39 Li, J., Zhang, B., Chen, Y., Zhang, J., Yang, H., Zhang, J., Lu, X., Li, G. \& Qin, Y. Styrene hydrogenation performance of Pt nanoparticles with controlled size prepared by atomic layer deposition. Catal. Sci. Technol. 5, 4218-4223 (2015).

40 Pan, M., Brush, A. J., Pozun, Z. D., Ham, H. C., Yu, W. Y., Henkelman, G., Hwang, G. S. \& Mullins, C. B. Model studies of heterogeneous catalytic hydrogenation reactions with gold. Chem. Soc. Rev. 42, 5002-5013 (2013).

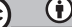

This work is licensed under a Creative Commons Attribution 4.0 International License. The images or other third party material in this article are included in the article's Creative Commons license, unless indicated otherwise in the credit line; if the material is not included under the Creative Commons license, users will need to obtain permission from the license holder to reproduce the material. To view a copy of this license, visit http://creativecommons.org/licenses/by/4.0/

Supplementary Information accompanies the paper on the NPG Asia Materials website (http://www.nature.com/am) 